\title{
CORNUBITE, $\mathrm{CU}_{5}\left(\mathrm{AsO}_{4}\right)_{2}(\mathrm{OH})_{4}$, FIRST OCCURRENCE OF SINGLE CRYSTALS, MINERALOGICAL DESCRIPTION AND CRYSTAL STRUCTURE
}

\author{
EKKEHART TILLMANNS; WOLFGANG HOFMEISTER and KLAUS PETITJEAN
}

TILLMANNS, EKKEHART; HOFMEISTER, WOLFGANG and PETITJEAN, KLAUS, 1985: Cornubite, $\mathrm{Cu}_{5}\left(\mathrm{AsO}_{4}\right)_{2}(\mathrm{OH})_{4}$, first occurrence of single crystals, mineralogical description and crystal structure. Bull. Geol. Soc. Finland 57, Part $1-2,119-127$.

The first single crystals of the rare copper arsenate hydroxide cornubite, $\mathrm{Cu}_{5}$ $\left(\mathrm{AsO}_{4}\right)_{2}(\mathrm{OH})_{4}$, have been found from a silicified barite vein at Reichenbach, Odenwald near Bensheim, Hessen, W.-Germany. Cornubite occurs as applegreen, translucent and mostly tabular crystals, up to $0.3 \times 0.3 \mathrm{~mm}$ in size and up to $0.05 \mathrm{~mm}$ thick. The lustre is vitreous, streak light-green and hardness 4 . The indices of refraction are between 1.8 and 1.9 and the optical angle is very large. The results of electron microprobe analysis are $\mathrm{As}_{2} \mathrm{O}_{5}$ 32.01, $\mathrm{CuO} 58.60, \mathrm{SiO}_{2} 0.34$, $\mathrm{P}_{2} \mathrm{O}_{5} 0.25, \mathrm{Al}_{2} \mathrm{O}_{3} 0.15, \mathrm{Fe}_{2} \mathrm{O}_{3} 0.32, \mathrm{H}_{2} \mathrm{O}$ (calculated) 5.27 weight \%, total 96.94 weight \%. The empirical formula is $\mathrm{Cu}_{5.03}\left(\mathrm{As}_{1.903} \mathrm{P}_{0.024} \mathrm{Fe}_{0.027} \mathrm{Al}_{0.020} \mathrm{Si}_{0.038}\right)_{2.013} \mathrm{O}_{8}$ $(\mathrm{OH})_{4}$. X-ray single crystal diffraction studies gave triclinic symmetry, space group Pī with $\mathrm{a}=6.121(1), \mathrm{b}=6.251(1), \mathrm{c}=6.790(1) \AA, \alpha=92.93(1)^{\circ}, \beta=$ $111.30(1)^{\circ}$ and $\gamma=107.47(1)^{\circ}, \mathrm{V}=227.09 \AA^{3}, \mathrm{Z}=1, \mathrm{D}_{\mathrm{x}}=4.85 \mathrm{Mg} / \mathrm{m}^{3}$. The crystal structure contains sheets of edge-sharing $\mathrm{CuO}_{6}$-octahedra // (011) connected by $\mathrm{AsO}_{4}$ tetrahedra via common corners and by hydrogen bonds.

Key words: cornubite, copper arsenate hydroxide.

Ekkehart Tillmanns and Wolfgang Hofmeister: Institut für Geowissenschaften, Universität Mainz, Saarstr. 21, D-6500 Mainz, Federal Republic of Germany.

Klaus Petitjean: Mainzer Str. 118, 6520 Worms, Federal Republic of Germany.

\section{Introduction}

The rare copper arsenate hydroxide cornubite, $\mathrm{Cu}_{5}\left(\mathrm{AsO}_{4}\right)_{2}(\mathrm{OH})_{4}$, was first described by Claringbull, Hey and Davis (1959) from Wheal Carpenter in Cornwall, U.K., and was named after the Latin name for Cornwall, Cornubia. It is dimorphous with cornwallite. Since the mineral occurred only as botryoidal fibrous masses, crystallographic data could not be de- termined, and the X-ray powder pattern could not be indexed although the fibre pattern led to the assumption of a triclinic unit cell with a cell volume of $228 \AA^{3}$ or an integral multiple of this. Cornubite has since been found at a number of localities together with copper arsenates or copper phosphates, e.g. at Neubulach (Walenta 1979) and Oberwolfach (Walenta 1975), Black Forest, W. Germany and at Imsbach, Donnersberg, W. Germany (Dreyer 1973), but the mineral 
has always formed crusts or fibrous masses; single crystals or aggregates of crystals have never been found. An X-ray powder investigation of apple-green, translucent, tabular crystals found in 1982 by one of the authors (K.P.) in a silicified barite vein on quartz with other secondary copper minerals near Reichenbach, Odenwald, W. Germany gave a powder pattern identical to that given by Claringbull et al. (1959) for cornubite (JCPDS \# 12-288). Reported here are the previously unknown data for this mineral.

\section{Occurrence}

The silicified barite vein at Reichenbach near Bensheim, Hessen, W. Germany, is the largest of a number of similar silicified veins occurring in the crystalline rocks of the northwestern
Odenwald area. Because of its peculiar pseudomorphic structures, the vein material is quarried in some places for use as ornamental stone. The vein strikes WNW and dips vertically to steeply southwards. Locally it reaches thicknesses of more than $10 \mathrm{~m}$ and can be traced in the field for about $5 \mathrm{~km}$, where it forms prominent cliffs (Teufelsstein, Borstein, Hohenstein, see Fig. 1).

The wallrocks of the vein are composed of various diorites and gabbros as well as orthogneisses and paragneisses. Owing to its mode of formation by replacement of barite the veinfilling quartz is not massive but displays an irregular pattern of crystallites of different shapes and sizes. According to Baier und Venzlaff (1961), this structure is due to quartz overgrowths on a previously formed framework of thin platy barite crystals. Subsequently barite dissolved and left open spaces. These casts and also the voids remaining between the former

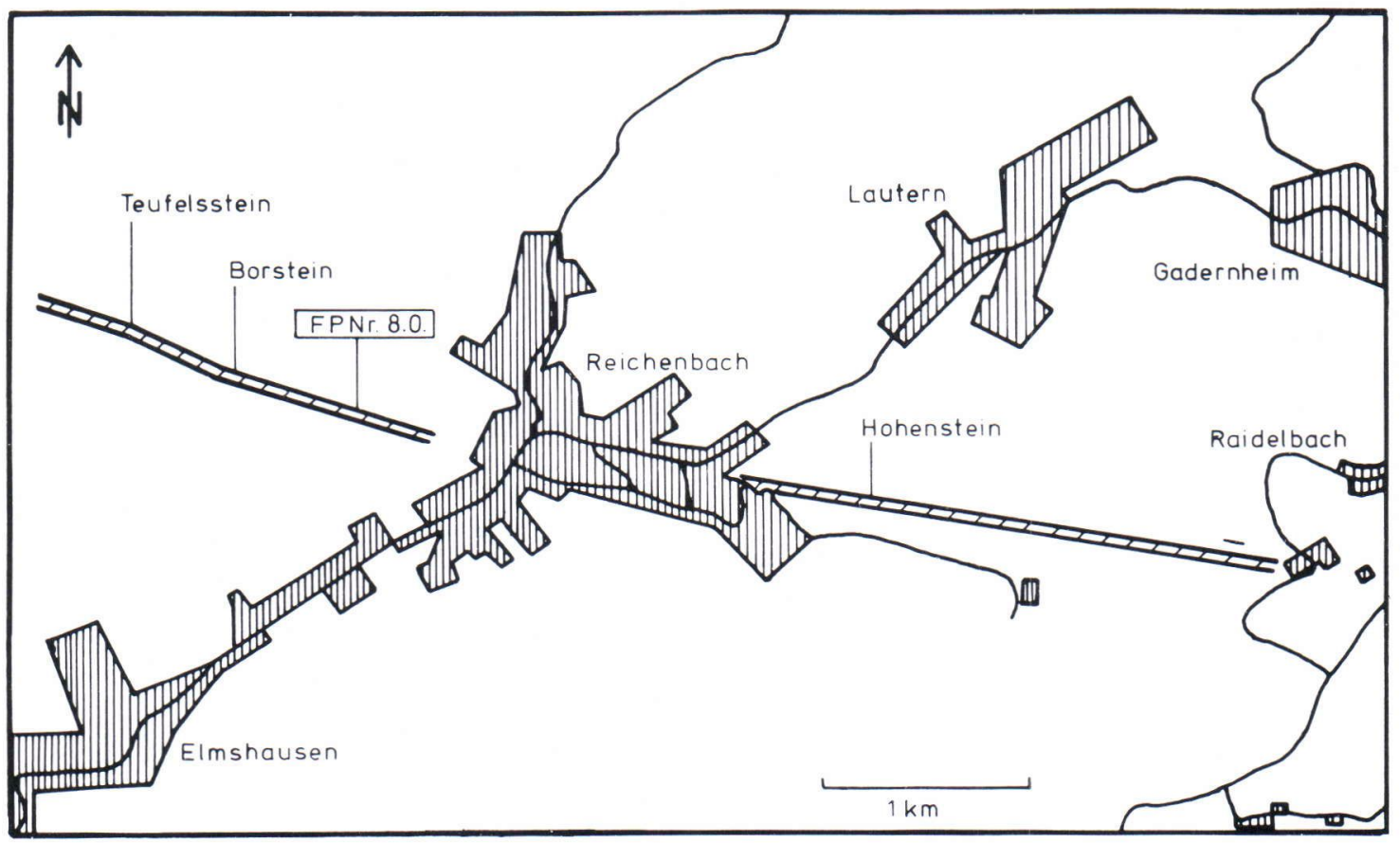

$\Longrightarrow$ Barite vein

Fig. 1. The silicified barite vein at Reichenbach. 
barite crystals were filled with quartz. The pseudomorphosed crystals show a preferred orientation perpendicular to the walls of the vein and occur in several generations separated by dense cryptocrystalline quartz. Small stringers of galena, chalcopyrite and minor fahlores are occasionally observed in the latter, whereas only isolated grains of ore minerals can be found within the former barite crystals. Under weathering conditions these primary ore minerals were partially oxidized, leading to a rich assemblage of secondary minerals (Table 1), predominantly phosphates, arsenates and vanadates. Redistribution and migration of metals during these oxidation reactions have caused secondarily formed minerals to occur as disseminations in adjacent parts of the vein, often well crystallized in open joints and drusy parts.

At the site designated FPNr8.0. in Fig. 1 (Borstein, map coordinates: r3477260 h5508500,

Table 1. Mineral phases of the silicified barite vein at Reichenbach.

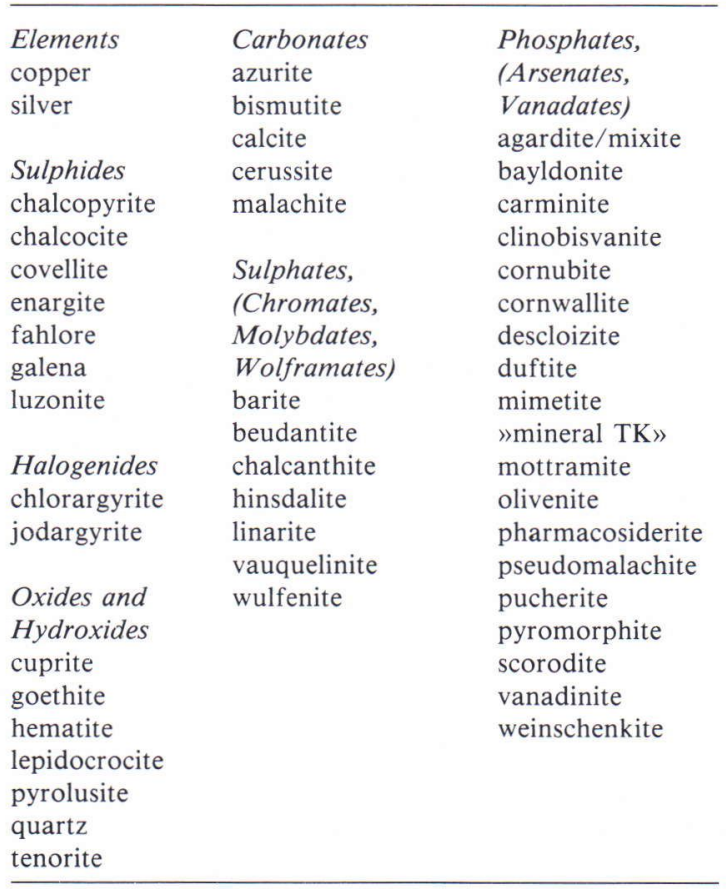

topographic map sheet 6218) some minor portions of the vein consist of a dense greyish quartz with abundant younger veinlets of crystalline quartz. Chalcopyrite is a primary mineral but has been largely decomposed into chalcocite and malachite. It was a block of this particular rock that contained the cornubite in clusters of acicular crystals together with goethite as velvetlike coatings on quartz; blue-green crystals of pseudomalachite; light-blue needle-like agardite/mixite crystals; dark-green tabular bayldonite crystals; lightgreen prismatic duftite crystals; colourless to bluish hinsdalite crystals and another not yet identified mineral, probably tsumcorite. The age relationships between the associated minerals are probably quartz goethite - cornubite + pseudomalachite agardite/mixite.

Crystals of cornubite are very rare at Reichenbach and only six specimens containing crystals of the mineral have been recovered so far.

Type material is preserved at the Naturhistorisches Museum, Mainz.

\section{Appearance and physical properties}

Cornubite from Reichenbach crystallizes as apple-green, translucent, mostly tabular crystals $/ /(2 \overline{1} 1)$ that are often irregularly intergrown. Individual platelets are up to $0.3 \times 0.3 \mathrm{~mm}$ in size and up to $0.05 \mathrm{~mm}$ thick. The lustre is vitreous, hardness is about 4 on Mohs' hardness scale and the streak is pale green. Two directions of cleavage, both perpendicular to (2ii1), can be observed: nearly parallel to (011) and (iīi) intersecting at an angle of about $70^{\circ}$.

Optical investigations were hampered by the high indices of refraction, by the morphology of the crystals and by the rapid reaction of the crystals with the immersion fluids. The refractive indices are between 1.8 and $1.9\left(\bar{n}_{\text {calc }}=\right.$ $1.854)$, the angle of the optical axes is very large, and dispersion has been observed with 
Table 2. Observed and calculated powder patterns for cornubite (*: Claringbull et al., 1959; + Debye-Scherrer diagram of a few single crystals from Reichenbach).

\begin{tabular}{|c|c|c|c|c|c|c|c|c|c|c|c|c|c|}
\hline${ }_{\mathrm{calc}}$ & ${ }^{\mathrm{I}} \mathrm{calc}$ & $h k l$ & $d_{\text {obs }}^{*}$ & $I_{\text {obs }}^{*}$ & $\begin{array}{c}+ \\
d_{\text {obs }}\end{array}$ & $\begin{array}{l}+ \\
\text { obs } \\
\end{array}$ & $d_{\text {calc }}$ & ${ }^{\mathrm{I}} \mathrm{calc}$ & $h \times 1$ & $d_{\text {obs }}{ }^{\star}$ & Iobs & $d_{\text {obs }}^{+}$ & $\begin{array}{l}+ \\
\text { I obs }\end{array}$ \\
\hline 5.36 & 17 & 100 & 5.35 & 60 & 5.34 & 30 & 1.566 & 2 & 023 & - & - & - & - \\
\hline 5.21 & 1 & $10 T$ & 5.22 & 10 & 5.21 & 10 & 1.563 & 1 & $2 \overline{3} 2$ & - & - & - & - \\
\hline 4.87 & 4 & $1 \div 0$ & 4.88 & 10 & - & - & 1.560 & 2 & 301 & - & - & - & - \\
\hline 4.71 & 100 & 011 & 4.72 & 100 & 4.69 & 80 & 1.544 & 3 & 113 & 1.544 & 10 & 1.539 & 30 \\
\hline- & $\bar{z}$ & $\bar{\tau}$ & 4.53 & 10 & - & - & 1.524 & 7 & 212 & & & - & - \\
\hline 4.30 & 7 & $1 T 1$ & 4.31 & 40 & 4.27 & 20 & 1.522 & 27 & $4 \pi \frac{2}{2}$ & 1.524 & 70 & 1.522 & 10 \\
\hline 3.93 & 4 & 011 & 3.93 & 20 & - & - & 1.518 & 1 & $13 \overline{3}$ & - & - & - & - \\
\hline 3.58 & 20 & $11 \bar{T}$ & 3.59 & 60 & 3.58 & 10 & 1.514 & 27 & 221 & 1.515 & 60 & 1.512 & 20 \\
\hline $\begin{array}{l}3.49 \\
3.44\end{array}$ & 48 & $1 \overline{1} 1$ & 3.49 & 80 & 3.48 & 40 & 1.506 & 1 & $30 \pi$ & - & - & - & - \\
\hline $\begin{array}{l}3.44 \\
3.32\end{array}$ & 3 & 101 & 3.42 & 10 & - & - & 1.501 & 3 & $3 \overline{1} \overline{4}$ & - & - & - & - \\
\hline $\begin{array}{l}3.32 \\
3.11\end{array}$ & 22 & $10 \overline{2}$ & 3.33 & 60 & 3.34 & 100 & 1.495 & 5 & $24 \pi$ & - & - & - & - \\
\hline $\begin{array}{l}3.11 \\
3.05\end{array}$ & 24 & 002 & 3.10 & 60 & 3.10 & 10 & 1.493 & 25 & 233 & 1.492 & 70 & - & - \\
\hline $\begin{array}{l}3.05 \\
2.980\end{array}$ & 21 & $1 \overline{2} 0$ & 3.05 & 60 & 3.04 & 10 & 1.492 & 5 & 421 & - & - & - & - \\
\hline $\begin{array}{l}2.980 \\
2.933\end{array}$ & $\begin{array}{l}24 \\
17\end{array}$ & 012 & 2.98 & 60 & 2.98 & 10 & 1.488 & 15 & $04 \pi$ & - & - & 1.488 & 20 \\
\hline 2.917 & $\begin{array}{r}11 \\
2\end{array}$ & $\begin{array}{lll}0 & 2 & 0 \\
2 & & 4\end{array}$ & 2.932 & 60 & 2.927 & 10 & 1.484 & 3 & 147 & - & - & - & - \\
\hline 2.868 & 23 & $\begin{array}{lll}2 & 0 & 1 \\
1 & 1 & 2\end{array}$ & - & - & - & - & 1.468 & 22 & $2 \frac{4}{1}$ & 1.467 & 50 & 1.465 & 40 \\
\hline 2.859 & 17 & $\begin{array}{lll}1 & 1 & 2 \\
0 & 2 & 1\end{array}$ & 2.868 & 70 & ${ }^{-} 860$ & $\overline{3}$ & $\begin{array}{l}1.459 \\
1.443\end{array}$ & $\begin{array}{l}4 \\
3\end{array}$ & $\begin{array}{lll}2 & \overline{2} & \overline{4} \\
2 & 3 & 1\end{array}$ & 1.457 & 10 & - & - \\
\hline 2.830 & 1 & $2 \frac{2}{1} 0$ & - & - & 2.860 & & $\begin{array}{l}1.443 \\
1.441\end{array}$ & $\begin{array}{l}3 \\
5\end{array}$ & $32^{2} \sum^{3} / 0^{1} 14$ & 1.442 & $20 \mathrm{~B}$ & - & $\overline{1}$ \\
\hline 2.697 & 15 & 121 & - & $\bar{z}$ & - & - & 1.421 & $\begin{array}{l}5 \\
2\end{array}$ & $\begin{array}{c}322 / 014 \\
329\end{array}$ & - & - & 1.439 & 20 \\
\hline 2.679 & 70 & 200 & 2.688 & 90 & 2.673 & 70 & 1.415 & 5 & $4 \Sigma 0 / 4 \overline{1} 0$ & - & - & 1.412 & 10 \\
\hline 2.624 & 1 & 111 & - & - & - & - & 1.409 & 2 & $40 \overline{3}$ & - & - & - & 10 \\
\hline 2.606 & 10 & 202 & 2.616 & 20 & - & - & 1.408 & 4 & $1 \overline{2} 4$ & 1.408 & 20 & - & - \\
\hline 2.564 & 12 & 012 & - & - & - & - & 1.407 & 1 & $3 \overline{2} \overline{4}$ & - & - & - & - \\
\hline 2.561 & 74 & $2 T \overline{2}$ & 2.562 & 100 & 2.554 & 60 & 1.399 & 3 & $3 \overline{4} \bar{T} / 1 \overline{1} 4$ & - & - & - & - \\
\hline 2.486 & $6 \overline{2}$ & $-\overline{2}$ & 2.525 & 10 & - & - & 1.394 & 3 & 203 & - & - & - & - \\
\hline $\begin{array}{l}2.486 \\
2.452\end{array}$ & $\begin{array}{r}62 \\
7\end{array}$ & $02 \frac{1}{2}$ & 2.489 & 100 & 2.478 & 50 & 1.391 & 2 & $2 \overline{4} \overline{2}$ & 1.392 & 10 & - & - \\
\hline 2.437 & 16 & $2 \overline{2} \overline{1}$ & - & - & - & - & 1.390 & 1 & $4 \overline{3} 1$ & - & - & - & - \\
\hline 2.402 & 7 & $\begin{array}{lll}2 & \overline{2} & 0 \\
1 & 1 & 2\end{array}$ & 2.443 & 40 & 2.440 & 20 & 1.375 & 3 & $4 \overline{3} 2$ & - & - & & - \\
\hline 2.377 & 11 & $12 \frac{1}{1}$ & 2.390 & $4 \overline{0}$ & 2.368 & $\overline{5}$ & $\begin{array}{l}1.373 \\
1.371\end{array}$ & $\begin{array}{r}3 \\
20\end{array}$ & $23^{0} 41^{1}$ & 1.373 & $6 \overline{0}$ & $-\overline{370}$ & 10 \\
\hline 2.356 & 11 & 022 & 2.358 & 50 & 2.308 & $\begin{array}{l}5 \\
-\end{array}$ & 1.352 & 1 & $1 \frac{1}{4} 2^{3}$ & 1.353 & $\begin{array}{l}60 \\
10\end{array}$ & 1.310 & - \\
\hline 2.355 & 6 & 211 & - & - & - & - & 1.341 & 3 & $3 \overline{4} 2$ & - & - & - & - \\
\hline 2.320 & 11 & 102 & - & - & - & - & 1.340 & 4 & $2 \overline{3} 3$ & 1.340 & 40 & - & - \\
\hline 2.304 & 33 & $2 T 1$ & 2.303 & 70 & 2.296 & 40 & 1.339 & 7 & $4 \overline{3} 0 / 400 / 123$ & - & - & 1.336 & 40 \\
\hline 2.266 & 3 & 120 & 2.265 & 20 & - & - & 1.336 & 2 & 302 & - & - & - & - \\
\hline 2.260 & 5 & $10 \overline{3}$ & - & - & - & - & 1.324 & 3 & $4 T \pi$ & 1.321 & 10 & - & - \\
\hline $\begin{array}{l}2.235 \\
2.222\end{array}$ & 9 & $21 \frac{2}{2}$ & 2.240 & 40 & 2.227 & 10 & 1.319 & 1 & 341 & - & - & - & - \\
\hline $\begin{array}{l}2.222 \\
2.167\end{array}$ & 2 & $1 \overline{2}$ & - & - & - & - & 1.312 & 2 & $2 \overline{1} \overline{5}$ & - & - & - & - \\
\hline $\begin{array}{l}2.167 \\
2.152\end{array}$ & 4 & 201 & 2.180 & 20 & - & - & 1.310 & 8 & 033 & 1.308 & 40 & 1.309 & 10 \\
\hline $\begin{array}{l}2.152 \\
2.147\end{array}$ & $\begin{array}{l}3 \\
4\end{array}$ & $2 \overline{2} \frac{2}{3}$ & - & - & - & - & 1.301 & 2 & $4 \cdot 1 \frac{3}{x}$ & - & - & - & - \\
\hline 2.137 & $\begin{array}{l}4 \\
3\end{array}$ & $11 \overline{3}$ & 2.143 & 40 & - & - & 1.299 & 6 & $4 \sqrt{3}$ & 1.300 & 50 & - & - \\
\hline 2.121 & $\begin{array}{l}3 \\
2\end{array}$ & $1 \overline{2} 2$ & - & - & - & - & 1.297 & 9 & $41 \overline{1}$ & - & - & 1.297 & 5 \\
\hline 2.091 & 25 & $\begin{array}{ll}22 & 2 \\
2 & 0\end{array}$ & - & - & 2.121 & 10 & 1.293 & 1 & 143 & - & - & - & - \\
\hline 2.080 & 5 & $20 \overline{3}$ & 2.090 & 60 & 2.083 & 30 & 1.282 & 3 & 024 & 1.281 & 20 & - & - \\
\hline 2.074 & 8 & $\begin{array}{lll}1 & 3 & 0 \\
0 & 0 & 3\end{array}$ & 2071 & $5 \overline{-}$ & - & - & 1.280 & 3 & $424 / 411$ & - & - & - & - \\
\hline 2.073 & 4 & $\begin{array}{lll}0 & 0 & \frac{3}{3} \\
1 & 1 & 3\end{array}$ & 2.071 & 50 & - & - & $\begin{array}{l}1.273 \\
1.269\end{array}$ & $\begin{array}{l}2 \\
1\end{array}$ & $\begin{array}{lll}2 & 1 & 3 \\
1 & 3 & 4\end{array}$ & 1250 & $\overline{10}$ & - & - \\
\hline & - & - & 2.027 & $10 B$ & - & - & 1.268 & 2 & 125 & 1.269 & $\begin{array}{r}10 \\
-\end{array}$ & 1.268 & 10 \\
\hline $\begin{array}{l}1.975 \\
1.969\end{array}$ & 5 & 112 & 1.973 & 40 & - & - & 1.256 & 1 & 114 & - & - & 1.258 & 5 \\
\hline $\begin{array}{l}1.969 \\
1.965\end{array}$ & 4 & 031 & - & - & 1.967 & 10 & 1.246 & 2 & $4 \overline{4} 1$ & - & - & - & - \\
\hline $\begin{array}{l}1.965 \\
1.955\end{array}$ & 2 & 022 & - & - & - & - & 1.239 & 3 & 225 & 1.241 & $10 \mathrm{~B}$ & - & - \\
\hline $\begin{array}{l}1.955 \\
1.951\end{array}$ & 22 & 030 & 1.957 & 60 & 1.950 & 20 & 1.228 & 2 & 025 & 1.227 & 20 & 1.227 & 5 \\
\hline $\begin{array}{l}1.951 \\
1.924\end{array}$ & 4 & $1 \overline{3} \overline{1}$ & $=$ & $=$ & $=$ & $=$ & 1.226 & 3 & $4 \overline{4} \overline{2}$ & - & - & - & - \\
\hline $\begin{array}{l}1.924 \\
1.918\end{array}$ & 7 & $2 \overline{3} 0$ & - & - & - & - & 1.225 & 2 & $41 \overline{4}$ & - & - & - & - \\
\hline $\begin{array}{l}1.918 \\
1.914\end{array}$ & 4 & $21 \overline{3}$ & - & - & - & - & 1.218 & 2 & 312 & - & - & - & - \\
\hline $\begin{array}{l}1.914 \\
1.911\end{array}$ & 4 & 321 & 1.919 & 40 & - & - & 1.217 & 2 & $25 \pi$ & 1.217 & 20 & - & - \\
\hline 1.911 & 2 & $30 \overline{2}$ & - & - & 1.911 & 20 & 1.216 & 2 & 141 & - & - & 1.216 & 5 \\
\hline 1.899 & 2 & $3 \overline{1} 0$ & 1.899 & 10 & - & - & 1.214 & 1 & 401 & - & - & - & - \\
\hline 1.897 & 3 & $2 \overline{3} 1$ & - & - & - & - & 1.208 & 1 & $3 \overline{2} \overline{5}$ & - & & - & - \\
\hline 1.859 & 1 & $02 \overline{3}$ & - & - & - & - & 1.205 & 7 & 214 & 1.203 & $40 B$ & - & - \\
\hline 1.853 & 4 & 013 & 1.853 & 10 & - & - & 1.199 & 3 & $5 \overline{2} \overline{1} / 33 \overline{1}$ & - & - & 1.195 & 5 \\
\hline 1.829 & 6 & $3 \overline{2} 0$ & 1.829 & 40 & - & - & 1.191 & 2 & 152 & 1.190 & 10 & - & - \\
\hline 1.822 & 5 & $22 \pi$ & - & - & - & - & 1.181 & 3 & $4 T 5 / 24 \overline{1}$ & 1.181 & 20 & - & - \\
\hline 1.814 & 3 & $2 T 2$ & - & - & 1.813 & 20 & 1.178 & 5 & $04 \overline{4} / 42$ 2 & - & - & 1.178 & 5 \\
\hline 1.809 & 1 & 032 & - & - & - & - & 1.175 & 6 & 0.15 & 1.172 & 20 & - & - \\
\hline 1.792 & 9 & 222 & 1.797 & 40 & 1.787 & 10 & 1.171 & 1 & $5 \overline{2}$ & - & - & - & - \\
\hline 1.760 & 1 & 132 & - & - & - & - & 1.169 & 1 & $05 \overline{2}$ & - & - & - & - \\
\hline 1.747 & 26 & 222 & 1.747 & 60 & 1.746 & 40 & 1.165 & 1 & $3 \overline{3} 3$ & - & - & - & - \\
\hline 1.737 & 7 & $30 \overline{3}$ & - & - & - & - & 1.162 & 1 & $13 \overline{5}$ & - & - & - & - \\
\hline 1.730 & 2 & $1 \overline{3} 1$ & - & - & - & - & 1.161 & 1 & $5 \overline{3} \overline{1}$ & - & - & - & - \\
\hline 1.722 & 3 & $2 \overline{3} \overline{2}$ & - & - & - & - & 1.157 & 2 & $14 \overline{4}$ & 1.158 & 10 & - & - \\
\hline 1.720 & 5 & 202 & 1.720 & 20 & 1.716 & 10 & 1.156 & 2 & 252 & - & - & - & - \\
\hline 1.718 & 1 & 103 & - & - & - & - & 1.152 & 8 & $4 \overline{2} 2$ & - & 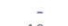 & 1.153 & 5 \\
\hline 1.694 & 3 & $31 \overline{2}$ & 1.696 & 10 & - & - & 1.151 & 6 & $441 / 234$ & 1.151 & 40 & - & - \\
\hline 1.662 & 5 & 130 & 1.662 & $40 B$ & 1.661 & 10 & 1.142 & 1 & $5 \overline{1} \overline{4}$ & - & - & - & - \\
\hline 1.660 & 4 & $3 \overline{3} \overline{1}$ & - & - & - & - & 1.137 & 2 & $1 \overline{2} 5$ & - & - & - & - \\
\hline 1.656 & 2 & $3 \overline{1} 1$ & - & - & - & - & 1.134 & 1 & 223 & - & - & - & - \\
\hline 1.629 & 1 & 321 & 1.630 & 10 & - & - & 1.130 & 5 & $20 \overline{6}$ & - & - & - & - \\
\hline 1.608 & 5 & $2 \frac{1}{4}$ & 1.608 & 10 & - & - & 1.124 & 3 & $235 / 5 T 0$ & - & - & - & - \\
\hline 1.591 & 18 & $21 \frac{1}{4}$ & 1.593 & 50 & 1.590 & 10 & 1.117 & 2 & 051 & - & - & - & - \\
\hline 1.589 & 3 & 313 & - & - & - & - & 1.113 & 1 & 504 & - & - & - & - \\
\hline 1.584 & 2 & $3 \overline{3} \overline{2}$ & - & - & - & - & 1.112 & 1 & 153 & - & - & - & - \\
\hline 1.582 & 4 & $1 \overline{4}$ & - & - & - & - & 1.110 & 1 & $05 \overline{3}$ & - & - & - & - \\
\hline 1.576 & 26 & 014 & 1.575 & 70 & - & - & 1.101 & 2 & $2 \overline{3} \frac{5}{5}$ & - & - & - & - \\
\hline 1.571 & 15 & 033 & - & - & 1.572 & 10 & 1.099 & 5 & $4 \overline{5} \overline{1}$ & - & - & 1.097 & 10 \\
\hline
\end{tabular}


red $>$ blue. For lack of material, the density could not be determined experimentally, but the calculated density is $4.85 \mathrm{Mg} / \mathrm{m}^{3}$; the density given by Claringbull et al. (1959) for cryptocrystalline material is $4.64 \mathrm{Mg} / \mathrm{m}^{3}$.

An analysis by electron microprobe (Cameca Camebax, MISO program (Abraham 1970)) with $20 \mathrm{kV}, 15 \mathrm{nA}$ gave $\mathrm{As}_{2} \mathrm{O}_{5}$ 32.01, $\mathrm{CuO}$ 58.60, $\mathrm{SiO}_{2} 0.34, \mathrm{P}_{2} \mathrm{O}_{5} 0.25, \mathrm{Al}_{2} \mathrm{O}_{3} 0.15, \mathrm{Fe}_{2} \mathrm{O}_{3}$ $0.32, \mathrm{H}_{2} \mathrm{O}$ (calc) 5.27 weight $\%$, total 96.64 weight $\%$, as the average of 20 measurements on a single crystal of cornubite. This leads to the empirical formula $\mathrm{Cu}_{5.03}\left(\mathrm{As}_{1.903} \mathrm{P}_{0.024} \mathrm{Fe}_{0.027}\right.$ $\left.\mathrm{Al}_{0.020} \mathrm{Si}_{0.038}\right)_{2.013} \mathrm{O}_{8}(\mathrm{OH})_{4}$ on the basis of $20 \mathrm{neg}$ ative charges ( $8 \mathrm{O}$ and $4 \mathrm{OH})$. The standards were $\mathrm{SrCuSi}_{4} \mathrm{O}_{10}, 2 \mathrm{Fe}_{2} \mathrm{O}_{3} \cdot \mathrm{As}_{2} \mathrm{O}_{5}, \mathrm{Al}_{2} \mathrm{O}_{3}, \mathrm{SiO}_{2}$, $\mathrm{AlPO}_{4}$ and $\mathrm{Fe}_{3} \mathrm{O}_{4}$. The idealized formula $\mathrm{Cu}_{5}$ $\left(\mathrm{AsO}_{4}\right)_{2}(\mathrm{OH})_{4}$ was used for all structural calculations.

Peak-search and indexing routines on an automatic 4-circle X-ray diffractometer and subsequent precession photographs yielded a triclinic unit cell, space group $\mathrm{P} \overline{1}$ with $\mathrm{a}=$ $6.121(1), \mathrm{b}=6.251(1), \mathrm{c}=6.790(1) \AA, \alpha=$ 92.93(1) ${ }^{\circ}, \beta=111.30(1)^{\circ}, \gamma=107.47(1)^{\circ}, \mathrm{V}$ $=227.09 \AA^{3}, Z=1$. The lattice constants were refined from the settings of 25 reflections with $24^{\circ} \leq \theta \leq 29^{\circ}$ which had been centred

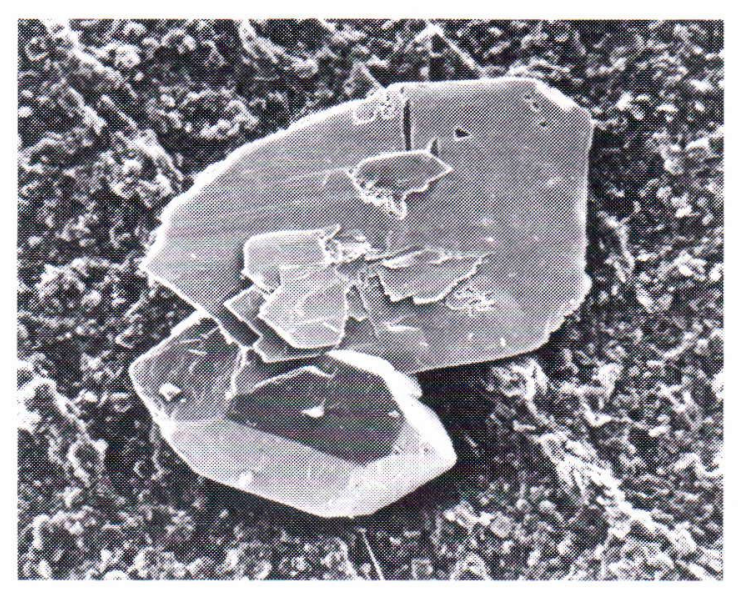

Fig. 2. Cornubite crystal, tabular // (2ī1) with quartz crystal (crystal size: $0.3 \times 0.2 \mathrm{~mm}$ ). on the diffractometer ( $\mathrm{MoK}_{\alpha}$ radiation). Table 2 contains the observed powder patterns of cornubite from Cornwall (Claringbull et al. 1959) and from Reichenbach as well as a pattern calculated from the refined atomic coordinates. The differences in the estimated intensity observations may be due to the small number of crystals from Reichenbach available for a DebyeScherrer diagram.

The scanning electron microscope photograph in Fig. 2 shows a cornubite single crystal of typical shape intergrown with a quartz crystal.

\section{Determination of the crystal structure}

The intensities of 2982 reflections with $\theta<40^{\circ}$ were measured on an automatic 4-circle diffractometer (Enraf-Nonius CAD-4) with monochromatized $\mathrm{MoK}_{\alpha}$ radiation, $\omega / 2 \theta$ step scan mode and a scan width of $\Delta \omega=0.60+0.5 \tan \theta$. The intensity and orientation of three standard reflections were monitored regularly. The instability of the measurement (Stout and Jensen 1968) was less than 0.006 for the three reference reflections. The data were corrected for Lorentz polarization and absorption effects. The crystal used for data collection was tabular // (2ii1) with approximate dimensions of $0.3 \times 0.2 \times 0.01$ $\mathrm{mm}$. The linear absorption coefficient was $18.85 \mathrm{~mm}^{-1}$ and the transmission factors were between 0.800 and 0.076 . Averaging of equivalent reflections gave 2809 unique reflections, 383 of which were considered to be unobserved $\left(\mathrm{I}<2 \sigma_{\mathrm{I}}\right)$.

The positions of $\mathrm{Cu}$ and $\mathrm{As}$ atoms were determined from the Patterson synthesis. Several cycles of Fourier and difference syntheses gave a model of the structure which could be refined with least-squares methods to a residual of 0.033 for 2436 observed structure factors. The R-value for all 2809 reflections was 0.042 . Atomic scattering factors and correction factors for anomalous dispersion were taken from $\gg$ In- 
Table 3. Fractional atomic coordinates with the significant figures of the estimated standard deviations in parentheses and vibrational parameters (in $\AA^{2}$ : $\mathrm{B}(\mathrm{eq})=8 / 3 \pi^{\text {a }}$ trace $\mathrm{U}$ ).

\begin{tabular}{lcccc}
\hline atom & $\mathrm{X} / \mathrm{a}$ & $\mathrm{Y} / \mathrm{b}$ & \multicolumn{1}{c}{$\mathrm{Z} / \mathrm{c}$} & $\mathrm{B}(\mathrm{eq})$ \\
\hline $\mathrm{Cu}(1)$ & 0.0 & 0.0 & 0.0 & $0.54(1)$ \\
$\mathrm{Cu}(2)$ & $-0.00406(5)$ & $0.32402(5)$ & $0.33203(5)$ & $0.62(1)$ \\
$\mathrm{Cu}(3)$ & $0.50677(5)$ & $0.32154(5)$ & $0.32393(5)$ & $0.54(1)$ \\
$\mathrm{As}$ & $0.44718(4)$ & $0.17614(4)$ & $0.79809(4)$ & $0.48(1)$ \\
$\mathrm{O}(1)$ & $0.8327(3)$ & $0.6883(3)$ & $0.8602(3)$ & $0.56(5)$ \\
$\mathrm{O}(2)$ & $0.8386(4)$ & $0.9905(3)$ & $0.2147(3)$ & $0.71(5)$ \\
$\mathrm{O}(3)$ & $0.6034(4)$ & $0.0162(3)$ & $0.7386(3)$ & $0.74(5)$ \\
$\mathrm{O}(4)$ & $0.8383(3)$ & $0.3443(3)$ & $0.5329(3)$ & $0.59(5)$ \\
$\mathrm{O}(5)$ & $0.3818(4)$ & $0.3395(3)$ & $0.6067(3)$ & $0.67(5)$ \\
$\mathrm{O}(6)$ & $0.3650(4)$ & $0.6572(3)$ & $0.9623(3)$ & $0.78(5)$ \\
\hline
\end{tabular}

ternational Tables for X-ray Crystallography», Vol.IV (1974). A table of observed and calculated structure factors is available upon re- quest. The refined fractional atomic coordinates and thermal parameters are given in Table 3 . The computer programs used in the course of the work are the same as given by Tillmanns, Hofmeister and Baur (1983).

\section{Results and discussion}

The most prominent feature of the crystal structure of cornubite is the sheet of edgesharing $\mathrm{CuO}_{6}$ octahedra parallel to $(0 \overline{1} 1)$. Individual sheets are linked via common corners by $\mathrm{AsO}_{4}$ tetrahedra and by hydrogen bonds. Figure 3 shows a polyhedral representation of the crystal structure, and Figure 4 the arrangement of

Table 4. Interatomic distances $(\AA)$ and angles $\left({ }^{\circ}\right)$. The standard deviations of $\mathrm{O}-\mathrm{O}$ distances are less than $0.005 \AA$.

\begin{tabular}{|c|c|c|c|c|c|c|c|c|c|c|}
\hline $\mathrm{Cu}(1)$ & - & $\mathrm{O}(1)$ & $2 x$ & $1.911(2)$ & & $\mathrm{Cu}(3)$ & - & $\mathrm{O}(4)$ & $1.966(1)$ & \\
\hline $\mathrm{Cu}(1)$ & - & $\mathrm{O}(2)$ & $2 x$ & $2.031(1)$ & & $\mathrm{Cu}(3)$ & - & $O(1)$ & $1.978(1)$ & \\
\hline $\mathrm{Cu}(1)$ & - & $O(3)$ & $2 x$ & $2.467(1)$ & & $\mathrm{Cu}(3)$ & - & $O(3)$ & $1.984(2)$ & \\
\hline $\mathrm{O}(1)$ & - & $\mathrm{O}(1)$ & & 3.822 & $180.0(1)$ & $\mathrm{Cu}(3)$ & - & $O(5)$ & $1.994(2)$ & \\
\hline $\mathrm{O}(1)$ & - & $O(2)$ & $2 x$ & 2.966 & $97.5(1)$ & $\mathrm{Cu}(3)$ & - & $\mathrm{O}(5)$ & $2.320(2)$ & \\
\hline $\mathrm{O}(1)$ & - & $O(2)$ & $2 x$ & 2.600 & $82.5(1)$ & $\mathrm{Cu}(3)$ & - & $O(6)$ & $2.342(2)$ & \\
\hline $\mathrm{O}(1)$ & - & $\mathrm{O}(3)$ & $2 x$ & 2.812 & $78.8(1)$ & $\mathrm{O}(4)$ & - & $O(1)$ & 3.938 & $173.9(1)$ \\
\hline $\mathrm{O}(1)$ & - & $O(3)$ & $2 x$ & 3.402 & $101.2(1)$ & $\mathrm{O}(4)$ & - & $O(3)$ & 2.843 & 92.1(1) \\
\hline $\mathrm{O}(2)$ & - & $\mathrm{O}(2)$ & & 4.062 & $180.0(1)$ & $\mathrm{O}(4)$ & - & $O(5)$ & 2.735 & $87.4(1)$ \\
\hline $\mathrm{O}(2)$ & - & $\mathrm{O}(3)$ & $2 x$ & 3.059 & $85.1(1)$ & $\mathrm{O}(4)$ & - & $\mathrm{O}(5)$ & 3.004 & $88.6(1)$ \\
\hline $\mathrm{O}(2)$ & - & $\mathrm{O}(3)$ & $2 x$ & 3.327 & $94.9(1)$ & $\mathrm{O}(4)$ & - & $O(6)$ & 3.131 & $92.9(1)$ \\
\hline \multirow[t]{2}{*}{$O(3)$} & - & $\mathrm{O}(3)$ & & 4.935 & $180.0(1)$ & $\mathrm{O}(1)$ & - & $\mathrm{O}(3)$ & 2.812 & $90.4(1)$ \\
\hline & & & & & & $O(1)$ & - & $O(5)$ & 2.808 & $90.0(1)$ \\
\hline $\mathrm{Cu}(2)$ & - & $\mathrm{O}(4)$ & & $1.954(1)$ & & $\mathrm{O}(1)$ & - & $\mathrm{O}(5)$ & 2.930 & $85.6(1)$ \\
\hline $\mathrm{Cu}(2)$ & - & $\mathrm{O}(1)$ & & $1.960(1)$ & & $O(1)$ & - & $\mathrm{O}(6)$ & 3.136 & $92.7(1)$ \\
\hline $\mathrm{Cu}(2)$ & - & $\mathrm{O}(2)$ & & $1.994(1)$ & & $\mathrm{O}(3)$ & - & $\mathrm{O}(5)$ & 3.978 & $178.8(1)$ \\
\hline $\mathrm{Cu}(2)$ & - & $\mathrm{O}(4)$ & & $2.009(1)$ & & $\mathrm{O}(3)$ & - & $\mathrm{O}(5)$ & 3.192 & $95.4(1)$ \\
\hline $\mathrm{Cu}(2)$ & - & $\mathrm{O}(5)$ & & $2.390(1)$ & & $\mathrm{O}(3)$ & - & $\mathrm{O}(6)$ & 3.076 & $90.3(1)$ \\
\hline $\mathrm{Cu}(2)$ & - & $\mathrm{O}(6)$ & & $2.421(1)$ & & $\mathrm{O}(5)$ & - & $\mathrm{O}(5)$ & 2.881 & $83.4(1)$ \\
\hline $\mathrm{O}(4)$ & - & $\mathrm{O}(1)$ & & 3.914 & $177.6(1)$ & $\mathrm{O}(5)$ & - & $\mathrm{O}(6)$ & 3.098 & $90.9(1)$ \\
\hline $\mathrm{O}(4)$ & - & $\mathrm{O}(2)$ & & 3.013 & $99.5(1)$ & $\mathrm{O}(5)$ & - & $\mathrm{O}(6)$ & 4.655 & $174.1(1)$ \\
\hline $\mathrm{O}(4)$ & - & $\mathrm{O}(4)$ & & 2.515 & $78.8(1)$ & & & & & \\
\hline $\mathrm{O}(4)$ & - & $\mathrm{O}(5)$ & & 3.189 & $93.9(1)$ & As & - & $\mathrm{O}(6)$ & $1.674(2)$ & \\
\hline $\mathrm{O}(4)$ & - & $\mathrm{O}(6)$ & & 3.131 & $90.8(1)$ & As & - & $\mathrm{O}(3)$ & $1.700(1)$ & \\
\hline $\mathrm{O}(1)$ & - & $\mathrm{O}(2)$ & & 2.600 & $82.2(1)$ & As & - & $\mathrm{O}(5)$ & $1.701(2)$ & \\
\hline$O(1)$ & - & $\mathrm{O}(4)$ & & 3.029 & $99.5(1)$ & As & - & $\mathrm{O}(2)$ & $1.714(1)$ & \\
\hline$O(1)$ & - & $\mathrm{O}(5)$ & & 2.930 & $84.1(1)$ & $\mathrm{O}(6)$ & - & $\mathrm{O}(3)$ & 2.717 & $107.3(1)$ \\
\hline$O(1)$ & - & $\mathrm{O}(6)$ & & $3: 144$ & $91.1(1)$ & $\mathrm{O}(6)$ & - & $\mathrm{O}(5)$ & 2.765 & $110.0(1)$ \\
\hline$O(2)$ & - & $\mathrm{O}(4)$ & & 4.001 & $176.7(1)$ & $\mathrm{O}(6)$ & - & $\mathrm{O}(2)$ & 2.842 & $114.0(1)$ \\
\hline $\mathrm{O}(2)$ & - & $\mathrm{O}(5)$ & & 3.394 & $101.1(1)$ & $\mathrm{O}(3)$ & - & $\mathrm{O}(5)$ & 2.770 & 109.1(1) \\
\hline $\mathrm{O}(2)$ & - & $\mathrm{O}(6)$ & & 2.945 & 83.1(1) & $\mathrm{O}(3)$ & - & $\mathrm{O}(2)$ & 2.824 & $111.6(1)$ \\
\hline $\mathrm{O}(4)$ & - & $\mathrm{O}(5)$ & & 2.735 & $76.4(1)$ & $\mathrm{O}(5)$ & - & $\mathrm{O}(2)$ & 2.705 & $104.8(1)$ \\
\hline $\mathrm{O}(4)$ & - & $\mathrm{O}(6)$ & & 3.395 & $99.6(1)$ & & & & & \\
\hline $\mathrm{O}(5)$ & - & $\mathrm{O}(6)$ & & 4.802 & $173.1(1)$ & & & & & \\
\hline
\end{tabular}




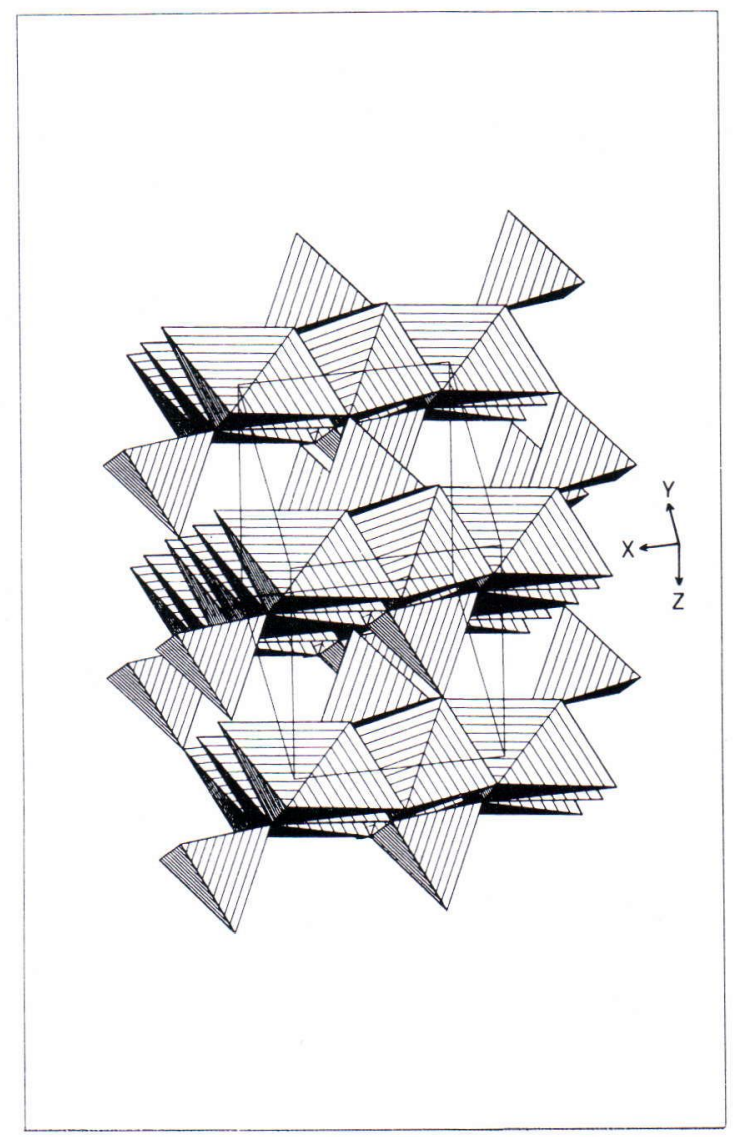

Fig. 3. Crystal structure of cornubite.
$\mathrm{CuO}_{6}$ octahedra within one sheet. Interatomic distances and angles are listed in Table 4.

All three crystallographically different copper atoms have a distorted octahedral or tetragonal bipyramidal coordination of $4+2$ oxygen atoms, as it is often found for coordination of oxygen atoms around copper (Zemann 1972). The arrangement of the four closer oxygen atoms is almost planar with values of $360^{\circ}$, $359.91^{\circ}$ and $359.89^{\circ}$ for the sums of the four $\mathrm{O}-\mathrm{Cu}-\mathrm{O}$ angles around $\mathrm{Cu}(1), \mathrm{Cu}(2)$ and $\mathrm{Cu}(3)$. The average $\mathrm{Cu}-\mathrm{O}$ distances in the plane are 1.971, 1.979 and $1.981 \AA$ respectively.

The $\mathrm{AsO}_{4}$ tetrahedra share all four corners with $\mathrm{CuO}_{6}$ octahedra, three in the same octahedral sheet and the fourth in an adjacent one. The spread of individual As-O distances is small, as are the deviations of tetrahedral angles from the ideal value of $109.47^{\circ}$.

An inspection of the bond strength sums received by each oxygen atom from the surrounding cations (Table 5) reveals that $\mathrm{O}(1)$ and $\mathrm{O}(4)$, which are coordinated by three $\mathrm{Cu}$ with a bond strength sum of 1.0 v.u., are the most likely $(\mathrm{OH})$-sites. Hydrogen bonds are probably formed from $\mathrm{O}(1)$ to an oxygen atom $\mathrm{O}(6)$, which is not in the same $\mathrm{CuO}_{6}$ octahedron as $\mathrm{O}(1)$, at a

Fig. 4. Sheet of $\mathrm{CuO}_{6}$ octahedra // (0I) in the crystal structure of cornubite.

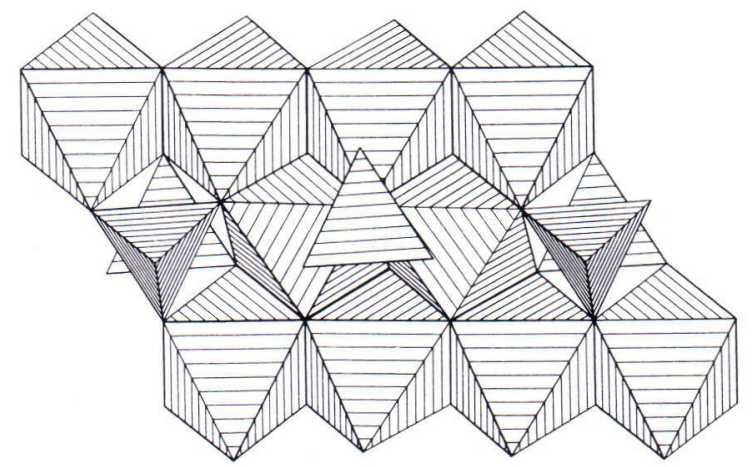


Table 5. Electrostatic bond strength sums (Baur 1970, Pauling 1960).

\begin{tabular}{lccccccc}
\hline atom & $\mathrm{Cu}(1)$ & $\mathrm{Cu}(2)$ & $\mathrm{Cu}(3)$ & $\mathrm{As}$ & $\mathrm{H}(\mathrm{D})$ & $\mathrm{H}(\mathrm{A})$ & $\Sigma$ \\
\hline $\mathrm{O}(1)$ & 0.33 & 0.33 & 0.33 & - & 0.83 & - & 1.83 \\
$\mathrm{O}(2)$ & 0.33 & 0.33 & - & 1.25 & - & - & 1.92 \\
$\mathrm{O}(3)$ & 0.33 & - & 0.33 & 1.25 & - & 0.17 & 2.09 \\
$\mathrm{O}(4)$ & - & $2 \times 0.33$ & 0.33 & - & - & - & 1.83 \\
$\mathrm{O}(5)$ & - & 0.33 & $2 \times 0.33$ & 1.25 & - & - & 2.25 \\
$\mathrm{O}(6)$ & - & 0.33 & 0.33 & 1.25 & - & 0.17 & 2.09 \\
\hline
\end{tabular}

distance of $2.709 \AA$, and from $\mathrm{O}(4)$ to $\mathrm{O}(3)$ at a distance of $2.865 \AA$.

$\mathrm{Cu}_{5}\left(\mathrm{AsO}_{4}\right)_{2}(\mathrm{OH})_{4}$ has been found in nature in two polymorphic forms, triclinic cornubite and monoclinic cornwallite. Three polymorphs are known for the analogous phosphate compound: the mineral pseudomalachite (Shoemaker, Anderson and Kostiner 1977) and two synthetic polymorphs: »PPM» (Anderson, Shoemaker, Kostiner and Ruszala 1977) and »QPM» (Shoemaker, Anderson and Kostiner 1981), which have also been found in nature (Shoemaker and Kostiner 1981). Two of these, pseudomalachite and the natural analog of "PPM», have also been identified in samples from Reichenbach.

Although similarities in X-ray diffraction patterns and lattice constants of cornwallite and pseudomalachite lead to the assumption that both minerals are isostructural, this is not true for cornubite and »QPM» even though both minerals crystallize in space group $\mathrm{P} \overline{1}$, have similar X-ray diffraction patterns and comparable unit cell volumes of $219.6 \AA^{3}$ and 227.1 $\AA^{3}$, respectively. The unit cell dimensions of "QPM» are $\mathrm{a}=4.445, \mathrm{~b}=5.873, \mathrm{c}=8.668 \AA$, $\alpha=103.62, \beta=90.35, \gamma=93.02^{\circ}$. Transformation of the unit cell of cornubite so that the $\mathrm{CuO}_{6}$ octahedral sheets are oriented the same way in both cells gives $\mathrm{a}=9.46(2 \times 4.73), \mathrm{b}=$ 6.12, $\mathrm{c}=8.99 \AA$ and $\alpha=119, \beta=85, \gamma=93^{\circ}$ (transformation matrix: $0 \overline{1} 1 / 1 / 100 / 011$ ). It is to this B-centred cell that the layer-line spacings derived by Claringbull et al. (1959) from a fibre photograph correspond.

Topological analyses of the crystal structures (Baur, Tillmanns and Hofmeister 1983) show that, while $\mathrm{CuO}_{6}$ octahedra are topologically identical in both structures, their connection is not. This results in a different arrangement of edge-sharing $\mathrm{CuO}_{6}$ octahedra within one sheet. A difference also exists in the way in which two octahedral sheets are linked by $\mathrm{XO}_{4}$ tetrahedra: in cornubite, the $\mathrm{AsO}_{4}$ tetrahedra share three corners with one sheet and the fourth with an adjacent one; in "QPM» the $\mathrm{PO}_{4}$ tetrahedra share two corners each with neighbouring sheets.

The structures can be expressed formally as close-packed arrangements of oxygen atoms with $\mathrm{Cu}$ in octahedral and $\mathrm{P}$ or As in tetrahedral voids. This arrangement, however, is appreciably distorted in the phosphate polymorphs, probably because of the smaller dimensions of the $\mathrm{PO}_{4}$ tetrahedron. Thus the pseudohexagonal angle in $" \mathrm{QPM} »$ is reduced to $104^{\circ}$ from its ideal value of $120^{\circ}$, whereas in cornubite it is $119^{\circ}$.

These distortions are also reflected in the shape of the coordination octahedra around $\mathrm{Cu}$, which are much more irregular in $» \mathrm{QPM} »$ than in cornubite.

Acknowledgements. The authors are indebted to Dr. K. Abraham and Dr. B. Schulz-Dobrick for the microprobe analyses and to N. Sieber, Dr. R. Krupp and Dr. O. Medenbach for useful discussions. Computing time was provided by the Computing Center of the University of Mainz. 


\section{References}

Abraham, K., 1970. MISO. University of Bochum, FRG. Anderson, J. B.; Shoemaker, G. L.; Kostiner, E. \& Ruszala, F. A., 1977. The crystal structure of synthetic $\mathrm{Cu}_{5}-$ $\left(\mathrm{PO}_{4}\right)_{2}(\mathrm{OH})_{4}$, a polymorph of pseudomalachite. Am. Mineral. 62, 115-121.

Baier, E. \& Venzlaff, V., 1961. Über die Verquarzung von Barytgängen. Notizbl. hess. L.-Amt Bodenforsch. 89, $365-379$.

Baur, W. H., 1970. Bond length variation and distorted coordination polyhedra in inorganic crystals. Trans. Amer. Cryst. Assoc. 6, 129-155.

Baur, W. H.; Tillmanns, E. \& Hofmeister, W., 1983. Topological analysis of crystal structures. Acta Cryst. B 39, 669-674.

Claringbull, G. F.; Hey, M. H. \& Davis, R. J., 1959. Cornubite, a new mineral dimorphous with cornwallite. Min. Mag. 32, 1-6.

Dreyer, G., 1973. Neue Mineralien der Rheinpfalz. Mitt. Pollichia III. Reihe, 20, 113-136.

International Tables for X-ray Crystallography, Vol IV. Revised and supplementary tables, 1974. (Ibers, I. A. and Hamilton, W. C., eds.). Birmingham, Kynoch Press.
Pauling, L., 1960. The Nature of the Chemical Bond. 3rd ed. Ithaca: Cornell Univ. Press.

Shoemaker, G. L.; Anderson, J. B. \& Kostiner, E., 1977. Refinement of the crystal structure of pseudomalachite. Am. Mineral. 62, 1042-1048.

Shoemaker, G. L.; Anderson, J. B. \& Kostiner, E., 1981. The crystal structure of a third polymorph of $\mathrm{Cu}_{5}$ $\left(\mathrm{PO}_{4}\right)_{2}(\mathrm{OH})_{4}$. Am. Mineral. 66, 169-175.

Shoemaker, G. L. and Kostiner, E., 1981. Polymorphism in $\mathrm{Cu}_{5}\left(\mathrm{PO}_{4}\right)_{2}(\mathrm{OH})_{4}$. Am. Mineral. 66, 176-181.

Stout, G. H. \& Jensen, L. H., 1968. X-ray Structure Determination. A Practical Guide. 1st printing. New York: The Macmillan Company.

Tillmanns, E.; Hofmeister, W. \& Baur, W. H., 1983. Crystal Structure of the Microwave Dielectric Resonator $\mathrm{Ba}_{2}$ $\mathrm{Ti}_{9} \mathrm{O}_{20}$. J. Am. Ceram. Soc. 66, 268-271.

Walenta, K., 1975. Die Sekundärmineralien des Schwerspatganges der Grube Clara bei Oberwolfach im mittleren Schwarzwald. Der Aufschluß 26, 369-411.

Walenta, K., 1979. Die Sekundärmineralien der Erzgänge von Neubulach im nördlichen Schwarzwald. Der Aufschluß 30, 213-252.

Zemann, J., 1972. Copper. Crystal Chemistry. Handbook of Geochemistry, Vol. II, (Wedepohl, K. H. ed.). Berlin: Springer-Verlag. 